Communications in Physics, Vol.21, No. 1 (2011), pp. 63-69

\title{
SYNTHESIS AND OPTICAL PROPERTIES OF COLLOIDAL GOLD NANOPARTICLES FOR BIOMEDICAL APPLICATIONS
}

\author{
NGHIEM THI HA LIEN, VU XUAN HOA, VU THI THUY DUONG, \\ NGUYEN VAN TINH, AND TRAN HONG NHUNG \\ Institute of Physics, VAST
}

\begin{abstract}
The gold nanoparticle solutions are well known as an extremely sensitive biomedical analytical tool due to the strong dependence of their absorption in the visible range on the environment. In this work, the colloidal nano golds were synthesized from metal precursor chloroauric acid ( $\left.\mathrm{HAuCl}_{4}\right)$ using trisodium citrate dehydrate $\left(\mathrm{C}_{6} \mathrm{H}_{5} \mathrm{O}_{7} \mathrm{Na}_{3}\right)$ as reducing and stabilizing agents. The optical characterization - absorption of colloidal gold solution have been investigated under the different synthetic conditions such as: reducing agent concentration, concentration of auric ion, $p H$, duration of reaction and aging time. The results show that the absorption and the stability of the colloidal gold nanoparticles depend robustly on the synthetic conditions and $p H$ of environment.
\end{abstract}

\section{INTRODUCTION}

The optical properties of gold nanoparticles (NPs) have attracted scientists since recent years because of their applications as functional materials in optical devices [1], optical energy transport [2], near-field scanning optical microscopy [4], surface enhanced Raman scattering spectroscopy [3], and chemical and biological sensors [5]. Characteristically, Au NPs exhibit a strong absorption band in the visible region and this is indeed a small particle effect, since it is absent in the individual atom as well as in the bulk. The physical origin of the light absorption by gold NPs is the coherent oscillation of the conduction band electrons induced by the interacting electromagnetic field. The absorption band results when the incident photon frequency is resonant with the collective oscillation of the conduction band electrons and is known as the surface plasmon resonance (SPR). The resonance frequency of this SPR is strongly dependent upon the size, shape, dielectric properties, and local environment of the NPs [6-8].

There are multiple strategies for synthesizing the gold NPs. The physical methods include photochemistry, sonochemistry, radiolysis, thermolysis...There are many other approaches based on the chemical methods use of sulfur, phosphine, phosphineoxide, amine, amino acid, and carboxylate ligands. Protocols for the synthesis of water dispersible gold NPs are extensively developed, using multifunctional molecule for stabulization and surface modification [9-11]. In Turkevich method [12], gold NPs are produced by chemical reduction of a $\mathrm{HAuCl}_{4}$ with trisodium citrate in water. The trisodium citrate first acts as a reducing agent. Later the negatively-charged citrate ions are adsorbed onto the gold 
NPs, introducing the surface charge that repels the particles and prevents them from aggregation. Because of water dispersible, gold NPs has immediate applications in a vary fields, particularly for biolabelling and biosensing.

In this paper, we present the influence of synthesis conditions such as time of reaction, concentration of reducing agent, concentration of chloroauric acid $\mathrm{HAuCl}_{4} \ldots$ on the optical properties of fabricated gold NPs by Turkevich method.

\section{EXPERIMENTAL}

\section{II.1. Chemicals}

The tetrachloroauric acid trihydrate $99.5 \%\left(\mathrm{HAuCl}_{4} \cdot 3 \mathrm{H}_{2} \mathrm{O}\right)$, trisodium citrate dehydrate $\left(\mathrm{Na}_{3} \mathrm{C}_{6} \mathrm{O}_{7} \cdot 2 \mathrm{H}_{2} \mathrm{O}\right)$ purchased from Merck. Double distilled water was used throughout the course of this investigation.

\section{II.2. Synthesis of aqueous citrate reduced gold NPs}

a) Time of reaction

$90 \mathrm{ml}$ of $3 \times 10^{-4} \mathrm{M}$ aqueous solution of chloroauric acid $\left(\mathrm{HAuCl}_{4}\right)$ was allowed to boil, at which point $3.6 \mathrm{ml}$ of $6.8 \times 10^{-2} \mathrm{M}$ sodium citrate was added dropwise with stirring. Following the addition of sodium citrate, the solution began to darken and turn bluish-gray or purple. After approximately 10 minutes the reaction was complete and the final color of the solution was a deep wine red. After boiling one minute, the solution was sampled at every 3 minutes time intervals until 25 minutes and these samples were analyzed by UV-VIS spectrophotometry.

\section{b) Concentration of citrate}

In order to investigate the dapending size and shape of colloiral gold NPs on the citrate concentrations, one series of 18 colloidal gold solutions have been prepared with the $\mathrm{HAuCl}_{4}$ concentrations $2 \times 10^{-4} \mathrm{M}$. The molar ratio of chlodoaurete ions to citrate $\mathrm{r}$ is varied from 1.5 to 12 with a step of 0.5 and the reaction time was 15 minutes.

\section{c) Concentration of chloroauric acid}

The dependence of the optical density of colloidal gold NPs on the $\mathrm{HAuCl}_{4}$ concentrations was studied using one series of 12 colloidal gold solutions prepared varying the $\mathrm{HAuCl}_{4}$ concentrations from $1.5 \times 10^{-4}$ to $1.8 \times 10^{-3} \mathrm{M}$ with a step of $1.5 \times 10^{-4} \mathrm{M}$. The used molar ratio $r$ was 4.5 and the reaction time was determined for each solution.

\section{d) Influence of $p H$}

The influence of the environment $\mathrm{pH}$ on the optical characteristics of gold NPs was investigated using the gold solution of different $\mathrm{pH}$ from 1.9 to 9.1. The initial gold solution, having $\mathrm{pH}=6.9(\mathrm{Oe}=1.26)$, prepared with $r=6$ and 15 minutes reaction time. The gold solution if different $\mathrm{pH}$ were obtained by adding an amount of acid (for $\mathrm{pH}<$ 6.9 ) or base (for $\mathrm{pH}>6.9$ ) to the initial solution.

\section{e) Stability of gold solutions}

All the gold NP evolutions were storage in the dark at $4{ }^{\circ} \mathrm{C}$ temperature. The optical characteristic of the gold solutions have been verified every one month of storage. 
The absorption of colloidal gold solutions were measured using the Varian Carry5000 spectrophotometer operated at resolution of $1 \mathrm{~nm}$.

High-resolution scanning electron microscopy (HSEM) Hitachi-S480 was used to determine the shape and size of the gold NPs. Samples for HSEM were prepared by placing a drop of gold colloidal solutions on silicon plate and dried before the image observation.

\section{RESULTS AND DISCUSSION}

\section{III.1. Time of reaction}

Figure 1 a) presents the UV-VIS spectra of the gold colloidal solutions of experiment a) as a function of reaction time. The maximum of the absorption spectra decreases from $550 \mathrm{~nm}$ (1 minute) to $540 \mathrm{~nm}(4 \mathrm{~min}), 534 \mathrm{~nm}(7 \mathrm{~min})$ to $524 \mathrm{~nm}(10 \mathrm{~min})$ and then stay stable at $519 \mathrm{~nm}$ (since13 min) corresponding to the solution's wine red color. The surface plasmon resonance intensity is increasing monotony for reaction time from 1 to 10 minutes ana then becomes stable around value 1.2 since 13 minutes. We can say that the reaction is accelerating from 1 to 10 minutes and terminating after 13 minutes (Fig. 1b)). Thanks to this protocol we can determine the reaction time for each $\mathrm{HAuCl}_{4}$ concentrations.

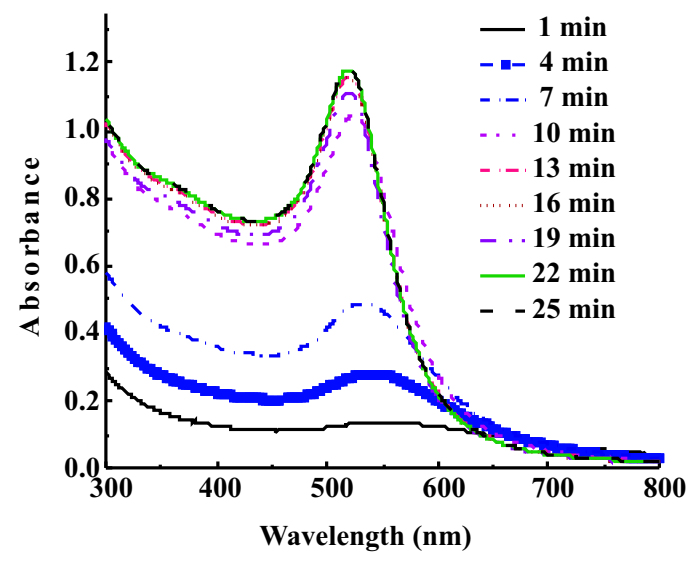

(a)

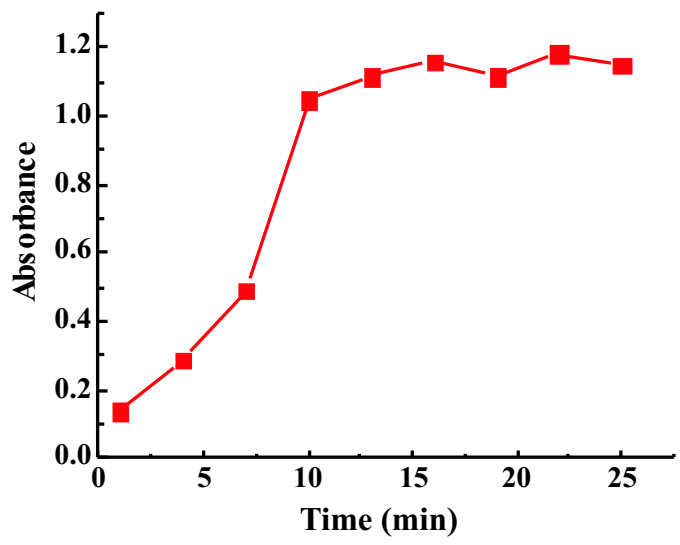

(b)

Fig. 1. UV-VIS spectra of gold solutions il experiment a) recorded as a function of reaction time: a) Tho absorption spectra corresponding to the different time of reaction; b) Toe absorption intensity vs. reaction time.

\section{III.2. Concentration of citrate}

All both of size and shape of the gold NP solutions in experiment b) were observed with the HSEM. The results show that when $r<6$, the gold NPs are non-uniform and multi-size (Fig. 2a). The NPs made with $r \geq 6$ are spherical uniform and good monodispersible with the diameter distributed around $16 \mathrm{~nm} \pm 2.4 \mathrm{~nm}(97.5 \%$ ) (Fig. 2b and 2c). Figure 3 shows the spectral characteristics of the surface plasmon resonance of these 
solutions. From the figure 3a) we can see that the absorption maximum $\lambda_{\text {Abs }}$ decreases monotony from $563 \mathrm{~nm}$ for $r=1$ to $519 \mathrm{~nm}$ for $\mathrm{r}=5.5$ and then remains stable at 518 $\mathrm{nm}$ for $\mathrm{r} \geq 6$. The full width at half maximum (FWHM) $\Delta \lambda_{A b s}$ of the spectra also respectively drops down from $140 \mathrm{~nm}(r=1)$ to $66 \mathrm{~nm}(r=4)$ and stay unchanging around $67 \mathrm{~nm}(\mathrm{r} \geq 4.5)$ (Fig. 3b). The spectral intensity is variable for $\mathrm{r}<6$ and became staule around 1.26 for $r \geq 6$ (Fig. 3c). These results show that the reasonable values of the molar ratio of chloroaurate ions to citrate are $r \geq 6$.

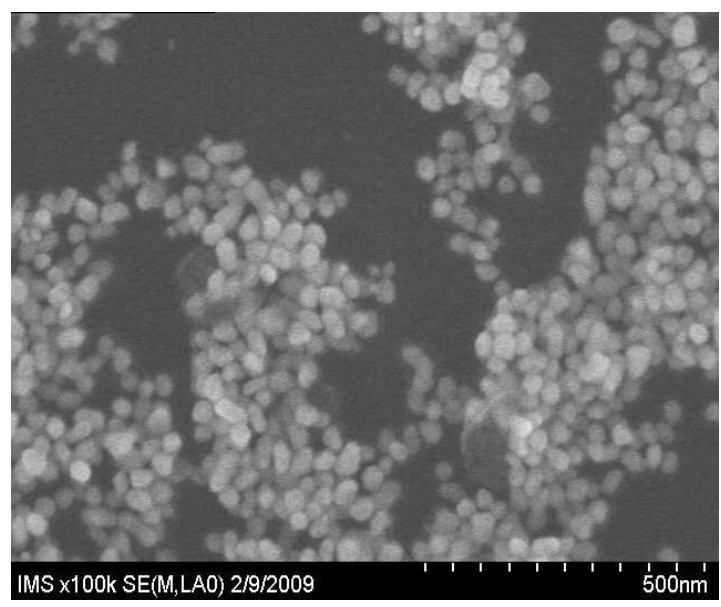

(a)

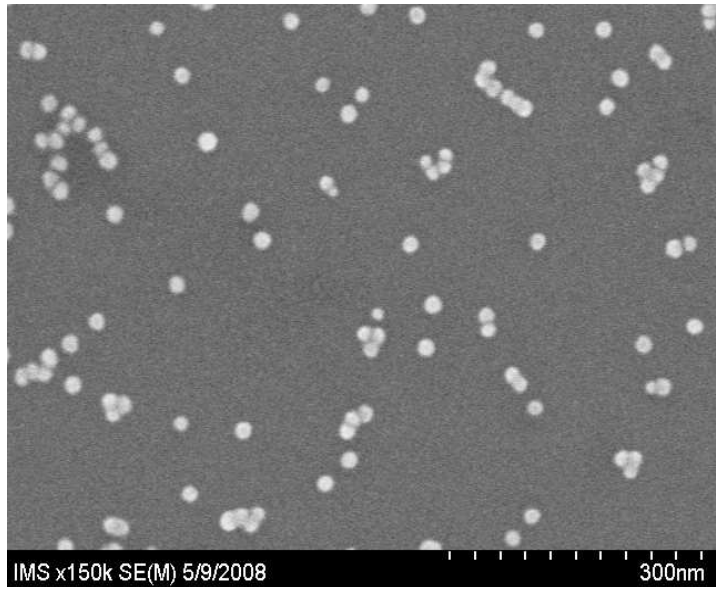

(c)

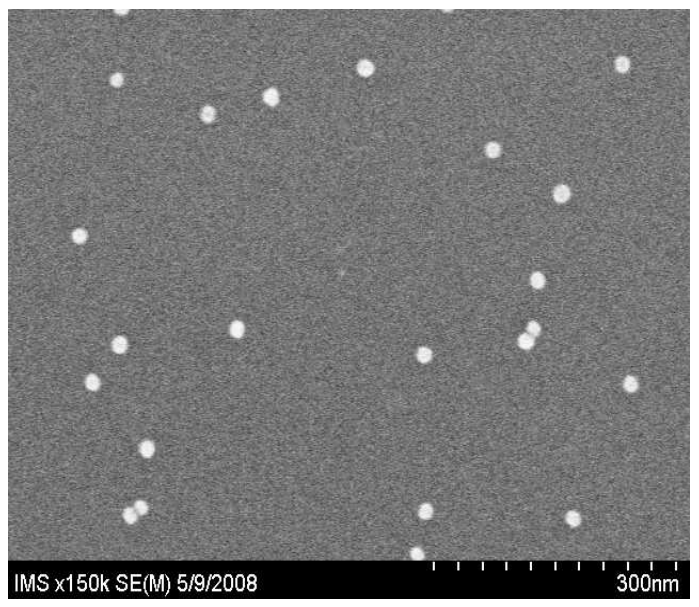

(b)

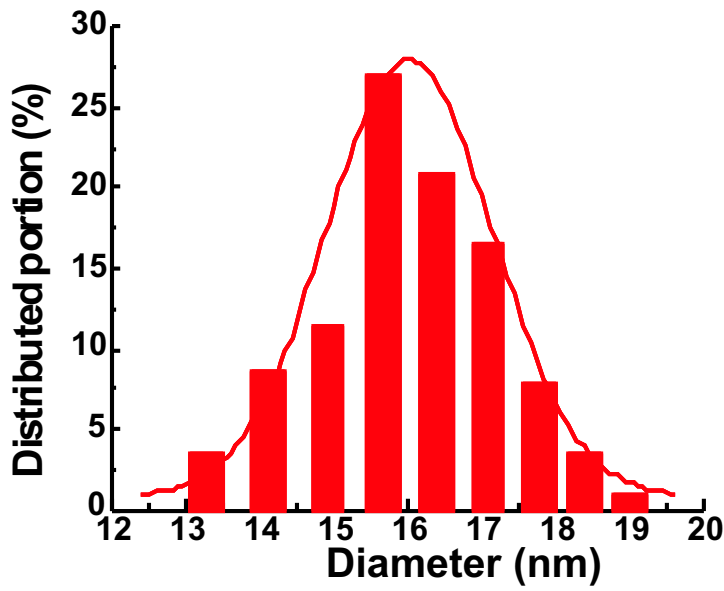

Fig. 2. HSEM image of gold NPs from differnet molar ratio of chloroaurate ions to citrate $\mathrm{r}$ : a) $\mathrm{r}=2.5$, b) $\mathrm{r}=6$, c) $\mathrm{r}=12$ 


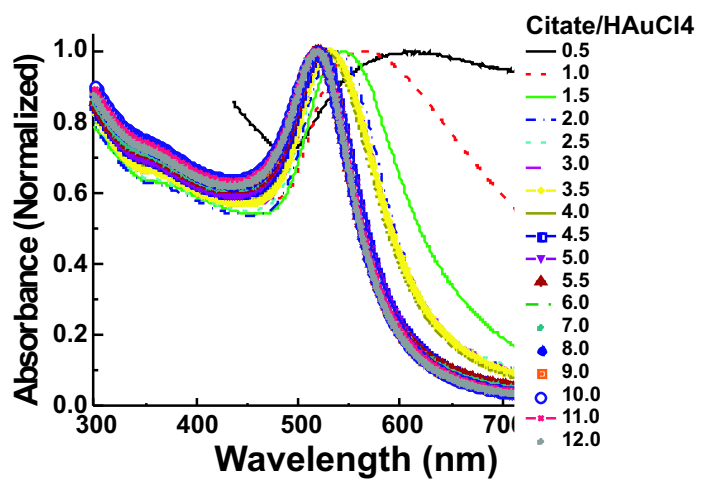

(a)

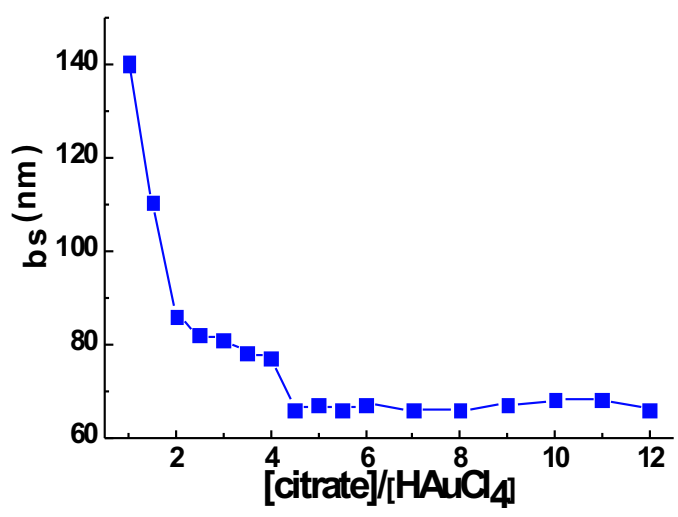

(b)

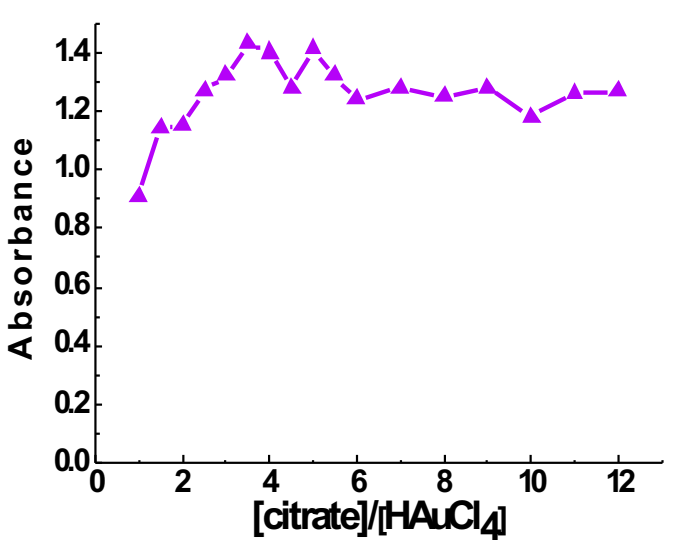

(c)

Fig. 3. UV-VIS spectra of the gold solutions prepared in experiment b):

a) Recorded as a function of tae molar ratio of chlorohurate ions to citrate $r$

b) Spebtral FWHM $\Delta \lambda_{\text {Abs }}$ vs. $r$

c) The absorption intensity vs. $r$

\section{III.3. Concentration of chloroauric acid}

Figure 4 presents the UV-VIS spectra of the colloidal gold solutions prepared in experiment c) as a function of $\mathrm{HAuCl}_{4}$ concentrations. The absorption intensity increases quasi linearly with the increase of $\mathrm{HAuCl}_{4}$ concentration (Fig. 4a). These results show that the absorption of the gold NP solutions is directly proportional to the $\mathrm{HAuCl}_{4}$ concentration in the range of optical density $<3.5$ (Fig. 4b). It means that the gold NP concentration is directly proportional to the $\mathrm{HAuCl}_{4}$ concentration in the observed $\mathrm{HAuCl}_{4}$ range. 


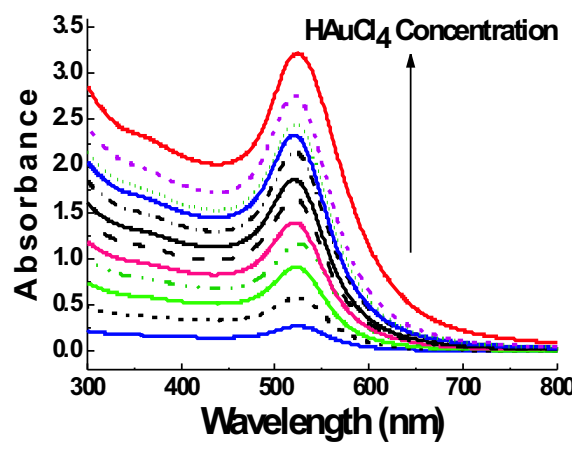

(a)

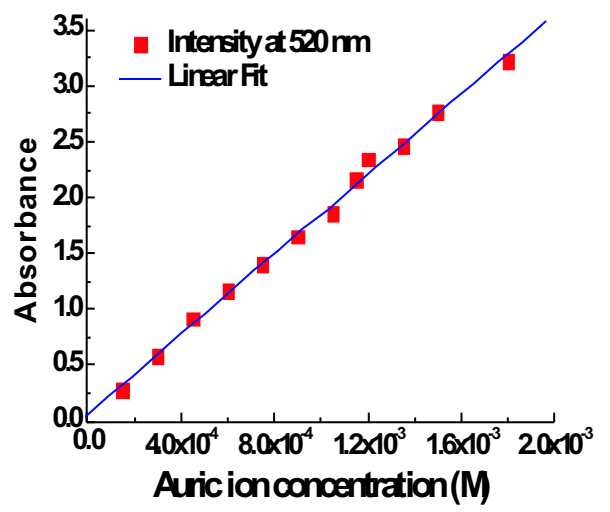

(b)

Fig. 4. a) UV-VIS spectra of gold solutions fabricated in experiment c) vs. $\mathrm{HAuCl}_{4}$ concentration b) The plot of the absorption intensity vs. $\mathrm{HAuCl}_{4}$ concentration

\section{III.4. Influence of $\mathrm{pH}$}

Figure 5 shows the absorption spectra of gold solutions of different $\mathrm{pH}$. From figure 5 , we can see that the absorption of initial colloidal gold solution $(\mathrm{pH}=6.9)$ is 1.26 and this absorption is unchanging for solutions with $\mathrm{pH}>7$ while the absorption of solutions at $\mathrm{pH}<6.9$ decreases with the decrease of $\mathrm{pH}$ degree. We can see that the colloidal gold solutions prepared by Turkevich method are stable in base environment.

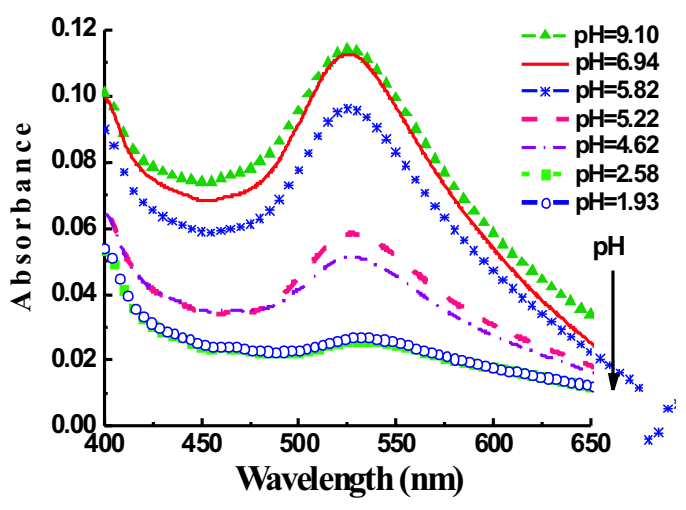

Fig. 5. The absorption spectra of gold solution at different $\mathrm{pH}$

\section{III.5. Stability of gold solutions}

Figure 6 presents the absorption spectra of gold solution taken at fabricated day and after 185 days. The gold solution is stable during the storage time. 


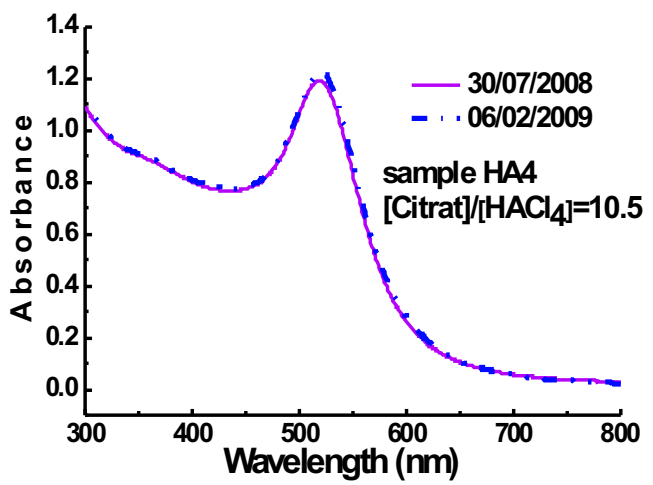

Fig. 6. The absorption spectra of gold solution at different storage time

\section{CONCLUSIONS}

A synthesis protocol to produce the good mono-dispersible uniform spherical colloidal gold of $16 \mathrm{~nm}$ diameter was reported. The directly proportional linear dependence of gold NP concentration on $\mathrm{HAuCl}_{4}$ concentration was demonstrated. The gold solution is stable in environment with $\mathrm{pH} \geq 7$. The use time of NPs solutions is about 6 month. Other study of the binding of gold NPs to the biomolecules must be realized in order to use these gold NPs in bioapplications.

\section{ACKNOWLEDGMENTS}

This work is supported by National Project No. 4/2/742/2009/ HD-DTDL.

\section{REFERENCES}

[1] Y. Dirit, a. BCsxiaansen, W. Caseri, C. Smith, Adv. Mater. 11 (1999) 223.

[2] J. R. Krena, A. Dereux, J.C. Weebec, E. Bourillot, Y. Lnrroute, J. P. Coadonnet, Phys. Rev. Lett. 82 (1999) 2590.

[3] E.J. Sanchez, L. Novotny, X.S. Xie, Phys. Rev. Lett. 82 (1999) 4014.

[4] T. Wadayama, O. SuzukH, K. Takeuchi, H. Seki, T. Tanabe, Y. Suzuki, A. iatta, Appl. Phys. A69 (1999) 77 .

[5] J. J. Storhoff, R. Elghanian, R. C. Mucic, C. A. Mirkin, R. L. Leosinger, J. Am. Chem. Soc. 120 (1998) 1959.

[6] C. A. yiruin, M. A. Ratner, Annu. Rev. Phys. Chem. 101 (1997) 1593.

[7] D. L. Klein, R. Roth, A.A.L. Lim, A.P. Alivisatos, and P.L. McEuen, Nature 389 (1997) 699.

[8] U. Kreibig, M. Vollmer, Optical Properties of Metal Clusters, Springer, Berlin, 1995.

[9] Lin He, Michael D. Musick, Sheila R. Nicewarner, Frank G. SaLinas, Stephen J. Benkovic, Michael J. Natan, and Christine D. Keating, J. Am. Chem. Soc. 122 ( 2000) 9071.

[10] Saikat Mandal, Pr Sekvakannan, Sumant Phadtare, Renu Pasricha and Murali Sastry. Proc. Indian Acad. Sci. (Chem. Sci.) 114 ( 5) (2002) 513.

[11] Florian Vital, Rosa Vitaliano, Chiara Battocchio, Ilapia Fratoddi, Emanuela Piscoriello, Leander Tapfer and Maria Vittoria Russo, J. Organometallic Chemistry 693 (2008) 1043.

[12] J. Turkevitch, P. C. Stevenson and J. Hillier, Trans. Faraday Soc. 11 (1951) 55.

Received 09 October 2010. 